\title{
Heart rate variability: An autonomic correlate of reaction time performance*
}

\author{
STEPHEN W. PORGES $\dagger$ \\ University of Illinois, Champaign, Ill. 61820
}

Resting level heart rate variablity was measured and correlated with subsequent reaction time performance in male college students. The Ss were required to respond as rapidly as possible following an extended visual warning signal of either fixed or variable duration. Resting level heart rate variability was related to reaction time only in the variable-foreperiod condition. The data are interpreted as illustrating the sensitivity of the relationship between heart rate variability and reaction time to slight changes in task demands.

Lacey \& Lacey (1958) hypothesized a relationship between spontaneous autonomic fluctuations and reaction time. They proposed that the frequency of spontaneous heart rate and skin resistance fluctuations would be correlated with reaction time performance. This hypothesis assumed that the autonomic afferents initiated by these spontaneous fluctuations would facilitate the cortical mechanisms associated with attentional processes.

In response to the Laceys' work, there have been reports of experiments relating spontaneous fluctuations in skin resistance (Rice, 1970; Wallace \& Fehr, 1970) and heart rate (Porges, 1972) to reaction time performance. This research has supported the Lacey and Lacey hypothesis by demonstrating that in adults and normal children greater fluctuations in skin resistance and heart rate were associated with faster reaction times.

In the present study, heart rate variability was used as a measure of spontaneous heart rate fluctuation. The study was designed to investigate the relationship between heart rate variability and reaction time performance in a simple reaction time task with an extended preparatory interval. Independent groups of Ss were tested under two different reaction time conditions, variable foreperiod or fixed foreperiod. The two foreperiod schedules were used to differentiate the predictive value of the resting heart rate variability under conditions of varying attentional demands. It was assumed that greater attentional demands were required when the termination of the foreperiod could not be anticipated.

\section{METHOD \\ Subjects}

Thirty-six male volunteers from introductory psychology courses received extra course credit for serving as Ss. As they appeared at the laboratory, they were assigned to the experimental conditions according to a predetermined random schedule.

*This work was supported in part by Grant MH-20329-01 from the National Institute of Mental Health.

+Address requests for reprints to Stephen W. Porges, Department of Psychology, University of Illinois, Champaign, Illinois 61820 .

\begin{abstract}
Apparatus
Stimuli were programmed by means of magnetic tape and were presented automatically. Reaction time was measured by a Standard electronic clock. The ambient noise level of the experimental room was approximately $50 \mathrm{~dB}$ (re .0002 dynes $\left(\mathrm{cm}^{2}\right.$ ). Room temperature was maintained at approximately $70^{\circ} \mathrm{F}$.

Heart rate was recorded on a Beckman Type RS dynograph at a paper speed of $5 \mathrm{~mm} / \mathrm{sec}$. The EKG recording sites were cleaned with $70 \%$ ethanol prior to the application of the electrodes. Zinc cup electrodes with a surface area of $3.14 \mathrm{sq} \mathrm{cm}$ and filled with cotton soaked in a $1 \% \mathrm{ZnSO}_{4}$ solution were used to record heart rate from EKG lead II. The heart rate was measured using a Beckman 9857 cardiotachometer.
\end{abstract}

\section{Procedure}

The Ss were randomly assigned to one of two groups of $18 \mathrm{Ss}$ each. The two groups were instructed to respond as rapidly as possible following the termination of the warning signal (green light) that remained illuminated for the duration of the foreperiod. Concurrent with the termination of the warning signal, the respond signal (red light) was illuminated. The respond signal was terminated by a buttonpress response.

The $S$ was seated in a comfortable armchair in a sound-attenuated room. After the pickups were attached, E read the instructions which informed $S$ of the task and then calibrated the recording equipment. Each $S$ then received two sessions of 10 reaction time trials, separated by a 2 -min rest period. The rest period was signaled to the Ss by the illumination of a white pilot light. The first session was used as practice to adapt the Ss to the experimental situation. The foreperiod duration was $16 \mathrm{sec}$ for the fixed-foreperiod group and varied among 16,22 , and $28 \mathrm{sec}$, according to a predetermined randomized schedule, for the variable PI group. Each group had the same schedule of intervals between the respond signal of one trial and the onset of the warning signal for the next trial. These intervals varied among 45,60 , and $75 \mathrm{sec}$, according to a predetermined randomized schedule. The latency between the termination of the warning signal and the buttonpress was recorded as S's reaction time.

\section{Quantification and Analysis of the Data}

One minute into the rest period, 25 interbeat intervals were measured. These $R-R$ intervals were transformed into heart rate by the cardiotachometer. Within each group the variance and the mean of the 25 beats were calculated and correlated with the mean reaction time performance of each $S$ during the second session.

The heart rate variance for each $S$ was ranked within each group. The $\mathrm{Ss}$ were divided into three equal-sized within-group levels of heart rate variance, i.e., high, mid, or low. Since the heart rate variability characteristic of the three levels was not equivalent for the two groups, the levels were nested under the factor of groups in a hierarchical repeated-measures analysis of variance on reaction time performance.

\section{RESULTS}

\section{Correlational Analyses}

Heart rate, heart rate variance, and mean reaction time were intercorrelated separately for the two groups. The only significant relationship was in the variable-foreperiod group, between the heart rate 


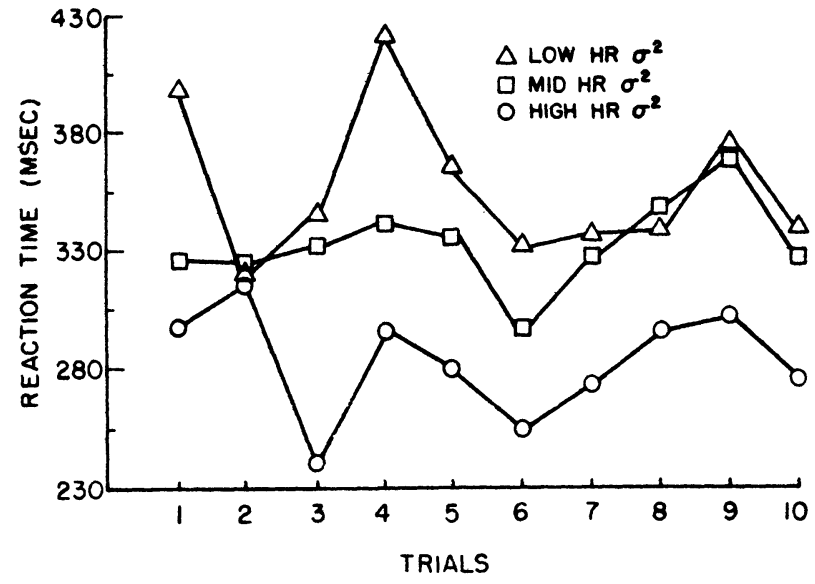

Fig. 1. Reaction time across trials for the three levels of heart rate variability in the variable-foreperiod condition.

variance and mean reaction time, $r(18)=-.70, p<.005$. This indicated that individuals exhibiting the larger heart rate variance during the resting period exhibited faster reaction times. This contrasted with the negligible -.03 correlation in the fixed-foreperiod group. A comparison of these two correlation coefficients indicated that the variable-foreperiod group exhibited a significantly greater concordance between heart rate variability and reaction time, $\mathrm{z}(30)=2.4, \mathrm{p}<.025$. Also of interest was the striking similarity between the two groups on the other comparisons, i.e., heart rate vs heart rate variance [VI, $\mathrm{r}(18)=.16 ; \mathrm{FI}, \mathrm{r}(18)=.11]$ and heart rate vs reaction time [VI, r(18) $=-.06 ; \mathrm{FI}, \mathrm{r}(18)=-.03$ ]

\section{Analyses of Variance}

A hierarchical analysis of variance with repeated measures was used to investigate the relationship between reaction time performance across trials as a function of the three resting level heart rate variability classifications (high, mid, low) within the two reaction time task demands (fixed, variable). The results indicated that the mean reaction time for the fixed-foreperiod condition $(271 \mathrm{msec})$ was significantly faster than the mean reaction time for the variable-foreperiod condition (321 msec), $F(1,30)=20.0, p<.0005$. When the two reaction time groups were pooled, reaction time did not differ as a function of level of heart rate variability.

The use of a hierarchical design does not generate a term to evaluate a possible Level of Heart Rate Variability by Reaction Time Task interaction, since any comparison utilizing level of heart rate variability would also be a function of the reaction time schedule to which the Ss were initially assigned. Thus, in a nested experiment, the only meaningful evidence for possible heart rate variability level effects on reaction time must be made from a comparison of heart rate variability level within reaction time task (Hays, 1963). Therefore, separate analyses of variance were calculated for each reaction time task.
The results indicated that, while there was no relationship between the heart rate variability classification and reaction time in the fixed-foreperiod condition, there was an inverse relationship between the rate variability classification and reaction time in the variable-foreperiod condition, $\mathrm{F}(2,15)=7.9, \mathrm{p}<.005$. This relationship, as illustrated in Fig. 1, indicated that the higher the heart rate variability classification the faster the reaction time. A Newman-Keuls test indicated that the mean of the reaction time performance was significantly greater for the high heart rate variability classification than for either the mid or low classifications, which did not differ. There were no trial effects or interactions.

\section{DISCUSSION}

The results suggest that under specific conditions resting level heart rate variability is related to reaction time. This relationship appears to be sensitive to the predictability of the respond signal, since heart rate variability was correlated with reaction time only in the variable-foreperiod group. It may be this type of attention, characterized by signal unpredictability, that is specifically related to autonomic lability.

In the present study superior performance was observed in the fixed-foreperiod group. This may have been facilitated by the predictability of the respond signal and the consequent development of temporal conditioned autonomic and motor responses. These effects have been previously demonstrated in fixed-foreperiod reaction time and classical conditioning experiments (Obrist, Webb, \& Sutterer, 1969; Porges, 1972).

Lacey \& Lacey (1958) suggested that the autonomic labile S could be characterized as being "hyperkinetic-impulsive." This would suggest that under conditions of signal unpredictability labile Ss would have difficulty inhibiting their responses. The present data, however, indicate that it is the labile $S$ who consistently had the faster reaction time across trials. This behavior would apparently necessitate an ability to suppress lability or inhibit the impulsivity to respond prior to foreperiod termination. In support of this notion, Porges (1972) has reported that Ss who have high pretrial heart rate variability and who tend to inhibit their lability by decreasing heart rate variability during a variable foreperiod also have the fastest reaction times.

The data for the variable-foreperiod condition support Lacey \& Lacey (1958), who have reported a statistically significant tendency for labile Ss to have faster reaction times. However, a survey of the literature reveals that experiments attempting to relate cardiac lability to reaction time performance have not always been successful. For example, Docter, Kaswan, \& Nakamura (1964) were able to replicate this relationship in one experiment but not in another. Moreover, Lacey and Lacey have, in subsequent unpublished replications, observed only consistently low and insignificant negative relationships between heart rate variability and reaction time. It is possible that these discrepancies are the result of the various types of tasks used to test reaction time.

The data from the present study gives relatively strong evidence of a relationship between heart rate variability and reaction time. The study illustrates the sensitivity of this relationship to any slight change in task demands, which may either enhance or mask the concordance between spontaneous heart rate fluctuations and reaction time.

\section{REFERENCES}

Docter, R. F. Kaswan, J. W., \& Nakamura, C. Y. Spontaneous heart rate and GSR changes as related to motor performance. Psychophysiology, 1964, 1, 73-78.

Hays, W. L. Statistics for psychologists. New York: Holt, Rinehart, \& Winston, 1963 
Lacey, J. I., \& Lacey, B. C. The relationship of resting autonomic activity to motor impulsivity. Proceedings of the Association for Research in Nervous \& Mental Disease, 1958, 36, 144-209.

Obrist, P. A., Webb, R. A., \& Sutterer, J. R. Heart rate and somatic changes during aversive conditioning and a simple reaction time task. Psychophysiology, 1969, 5, 696-723.

Porges, S. W. Heart rate variability and deceleration as indexes of reaction time. Journal of Experimental Psychology, 1972, 92, 103-110.

Rice, W. J. Reaction time correlates of spontaneous skin resistance responses and personality characteristics. Paper presented at the Tenth Annual Meeting of the Society for Psychophysiological Research, New Orleans, 1970.

Wallace, R. M.. \& Fehr, F. S. Heart rate, skin resistance, and reaction time of Mongoloid and normal children under baseline and distraction conditions. Psychophysiology, 1970, 6, 722-731.

(Received for publication January 15, 1973.) 